SERIES “CHRONIC THROMBOEMBOLIC PULMONARY HYPERTENSION"

\author{
Edited by J. Pepke-Zaba, M.M. Hoeper and M. Humbert
}

Number 3 in this Series

\title{
Surgical treatment of chronic thromboembolic pulmonary hypertension
}

\author{
David P. Jenkins*, Michael Madani" ${ }^{\#}$ Eckhard Mayer", Kim Kerr', Nic Kim, \\ Walter Klepetko ${ }^{\S}$, Marco Morsolini ${ }^{f}$ and Philippe Dartevelle**
}

ABSTRACT: It is likely that chronic thromboembolic pulmonary hypertension (CTEPH) is more prevalent than currently recognised. Imaging studies are fundamental to decision making with respect to operability. All patients with suspected CTEPH should be referred to an experienced surgical centre. Currently, there is no risk scoring stratification system to guide operability assessment and it is predominantly based on surgical experience.

The aim of pulmonary endarterectomy (PEA) is the removal of obstructive material to immediately reduce pulmonary vascular resistance. PEA affords the best chance of cure, but is difficult to perfect. Recognition and clearance of distal segmental and subsegmental disease is the main problem. The basic surgical techniques include: median sternotomy incision, cardiopulmonary bypass, arteriotomy incisions within pericardium, and a true endarterectomy with meticulous full distal dissection. Deep hypothermic circulatory arrest is recommended as the best means of reducing blood flow in the pulmonary artery to allow a clear field for dissection. In the recent PEACOG (PEA and COGnition) trial there was no evidence of cognitive impairment post-PEA.

Reperfusion pulmonary oedema and residual pulmonary hypertension are unique postoperative complications post-PEA and are associated with increased mortality. However, inhospital mortality is now $<5 \%$ in experienced centres.

KEYWORDS: Chronic thromboembolic pulmonary hypertension, deep hypothermic circulatory arrest, pulmonary artery, pulmonary endarterectomy, pulmonary vascular resistance, reperfusion lung injury

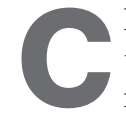

hronic thromboembolic pulmonary hypertension (CTEPH) remains under-diagnosed and carries a poor prognosis. The disease presents with nonspecific symptoms and general physicians may not be aware of the condition or the potential for treatment. Current medical treatment is, at best, palliative. Pulmonary endarterectomy (PEA) surgery offers the only chance of symptomatic and prognostic improvement and is curative in many patients with excellent long-term results.

It is likely that CTEPH is much more prevalent than recognised. If $3.8 \%$ of patients develop CTEPH following an acute pulmonary embolism [1], then there could be as many as 12,000 to 15,000 new cases annually in the USA alone, but only about 200 pulmonary endarterectomies are actually performed. Despite increased understanding, even after the diagnosis of CTEPH is established, operability assessment can still be challenging. Operability is based on the pre-operative estimate of surgical classification [2] and the pre-operative estimation of probable post-operative pulmonary vascular resistance (PVR), both of which determine risk of intervention and probable outcome. The surgical classification is an intra-operative assessment and even the best modern imaging can only estimate the probable type of disease found during surgery, and it is not possible to

\section{AFFILIATIONS}

*Dept of Cardiothoracic Surgery, Papworth Hospital, Cambridge, UK.

\#Division of Cardiovascular and Thoracic Surgery, University of California at San Diego, and +Division of Pulmonary and Critical Care Medicine, University of California at San Diego, San Diego, CA, USA.

"Dept of Thoracic Surgery, Kerckhoff Center, Bad Nauheim, Germany.

${ }^{\S}$ Dept of Thoracic Surgery, University of Vienna, Austria.

${ }^{f}$ Division of Cardiac Surgery, San Matteo Hospital, Pavia, Italy. ${ }^{* *}$ Dept of Thoracic and Vascular Surgery, Hopital Antoine Beclere, Paris, France.

CORRESPONDENCE

D.P. Jenkins

Papworth Hospital

Cambridge CB23 3RE UK

E-mail: david.jenkins@ papworth.nhs.uk

Received:

April 072012

Accepted after revision:

June 252012

First published online:

Nov 082012 
determine the ease of the dissection plane pre-operation. The final factor in operability assessment is the experience of the institution, including intensive care facilities, for post-operative care and most importantly the experience and skills of the operating surgeon.

Imaging studies are fundamental to decision making with respect to operability rather than merely the diagnosis of CTEPH. At this stage in patient assessment the ventilation/ perfusion $\left(V^{\prime} / Q^{\prime}\right)$ scan should not be forgotten and can be crucial for operability assessment by distinguishing between large and small vessel disease, as well as being the screening scan of choice [3]. In the former there are multiple unmatched segmental perfusion defects whereas in the latter there is a patchy mottled appearance. The $V^{\prime} / Q^{\prime}$ scan itself has $>96 \%$ sensitivity and $>90 \%$ specificity [3], but can underestimate the degree of obstruction, especially in web stenosis. Computed tomography (CT) pulmonary angiography scans are increasingly utilised for surgical assessment, as well as diagnosis in some centres [4]. The key features of CTEPH are dilated central pulmonary arteries, right ventricular enlargement, intraluminal thromboembolic material, enlarged bronchial arteries providing collateral blood flow, mosaic attenuation and parenchymal abnormalities secondary to infarcts. Two key rules are often misunderstood: 1) the absence of proximal clot on CT angiogram does not rule out surgically accessible chronic disease; and 2) the presence of central thrombus on CT does not confirm the diagnosis of CTEPH. Magnetic resonance imaging pulmonary angiograms are also increasingly used in many centres and can provide excellent three-dimensional views, but expert opinion remains divided as to whether they can replace conventional pulmonary angiograms. Conventional pulmonary angiography, usually combined with right (and left for coronary angiography if aged $>45$ yrs) heart catheterisation, remained the gold standard at the University of California at San Diego (UCSD; San Diego, CA, USA). It establishes the diagnosis and assesses the operative resectability. Two planes are important with lateral imaging improving anatomic definition especially in the lower lobes (fig. $1 \mathrm{a}$ and b). Right-sided pressures are obtained and the PVR is calculated. The risk of complications in experienced centres is very low. Pulmonary angioscopy is now rarely used in clinical practice.
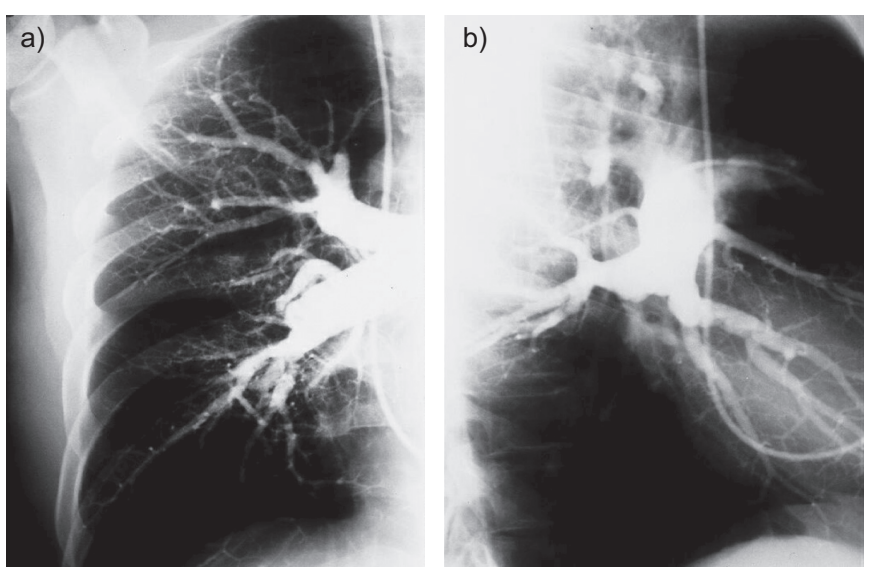

FIGURE 1. Pulmonary angiogram on a) anterior view and b) lateral view.
The final element of surgical selection is the balance of accessible clot with degree of pulmonary hypertension $(\mathrm{PH})$, in particular PVR. The effect of pre-operative medical therapy influencing haemodynamics is unknown. There are two key studies describing the association between: 1) pre-operative pulmonary resistance and in-hospital mortality [5]; and 2) post-operative residual PVR and in-hospital mortality [6]. There is no lower or higher limit for PVR, but $1,000 \mathrm{dyn} \cdot \mathrm{s}^{-1} \cdot \mathrm{cm}^{-5}$ clearly distinguishes those at higher and lower surgical risk. In the most recent cohort of patients at UCSD since 2006, in-hospital mortality was only $1.6 \%$ when PVR was $<1,000 \mathrm{dyn} \cdot \mathrm{s}^{-1} \cdot \mathrm{cm}^{-5}$ and $4.1 \%$ when it was $>1,000 \mathrm{dyn} \cdot \mathrm{s}^{-1} \cdot \mathrm{cm}^{-5}$. Other considerations include symptom status at rest and exercise and the patients' comorbid condition. In summary, all patients with suspected CTEPH should be referred to an experienced centre for confirmation of diagnosis and specifically an experienced surgical centre for assessment of operability. Consideration about commencing medical therapy for CTEPH patients should only occur following surgical assessment, as currently available drugs are not alternatives to PEA.

\section{ORGANISATION OF PEA SERVICES}

A PEA surgeon requires experience with $\mathrm{PH}$, technical expertise, and expertise with pre-operative evaluation and post-operative intensive care.

The PEA operation is difficult to learn and it is difficult to train new surgeons. Recognition and clearance of, especially, distal segmental and subsegmental disease is the main problem and before the threshold of competence is reached it is likely that at least 1 yrs training and/or experience of 100 cases is necessary. Surgical experience is the most important factor in achieving a good patient outcome.

A CTEPH centre needs extensive experience with cardiothoracic surgery, excellent pulmonary and cardiac services with emphasis on $\mathrm{PH}$ and excellent diagnostic imaging. As well as surgeons, experienced teams of radiologists, anaesthetists, intensivists, nurses, perfusionists and respiratory therapists are needed.

At the regional and national level the major question is how many surgical centres are necessary? Should the number of centres be based on the population, or on patients diagnosed, or number of cases performed? The actual incidence and prevalence are not known precisely and CTEPH is probably more prevalent than recognised. Recent calculations based on delegates attending the International Scientific and Educational Workshop in CTEPH (Cambridge, UK) meeting in June 2011 indicated that there were currently about 26 centres worldwide, but many have a low volume of cases. If it were possible to organise treatment facilities without limitation by local policy or geographical boundaries, the ideal plan might be one centre per 50 million population, geographically suited and performing $>75-100$ cases per year (perhaps minimum of 50 cases per year) to ensure excellent outcomes. For a new PEA centre, staff training could be helped by visiting a high-volume centre with a reciprocal visit by the host team to help with initial operations.

International networks could be important for improving communication and educating smaller centres to limit the learning curve of this demanding operation. 


\section{RISK SCORING FOR PEA SURGERY}

There cannot be a universal definition of operability, but a scoring system for evaluation of operative risk, morbidity and long-term outcome (PEA score) could be useful. Risk scoring systems such as Society of Thoracic Surgeons or EuroSCORE are based on huge datasets, that are not possible for PEA surgery. A major prognostic factor for PEA surgery is the experience of the surgeon and institution, as demonstrated by the CTEPH registry data [7]. Expert opinion consensus has concluded that major factors for any risk score system should include PVR, New York Heart Association class, 6-min walk distance, presence of indwelling catheters, medical pre-treatment and more subjective factors related to amount of disease on imaging studies. The only risk factors that impacted on inhospital mortality (univariate analysis) in the European CTEPH registry were presence of $\mathrm{PH}$-specific drug treatment, time from last pulmonary embolism, PVR value at diagnosis, 6-min walk distance at diagnosis and PVR at end of intensive care unit (ICU) stay [7].

However, many of the variables needed in a risk scoring system would be subjective, e.g. degree of segmental vessel involvement and clearability. Expert opinion has agreed that an international database (with prospective data collection) including risk factors and early and late outcome of PEA is necessary to increase knowledge. Non-operated CTEPH patients should also be included. An international surgical CTEPH network working via an appropriate platform would be necessary to affect this database. The number of centres per country should be limited (as in the UK) to exclude surgical experience as a risk factor for patient outcome. There is divided opinion on the value of a risk scoring system; it would allow comparison between centres, but there are concerns that it might reduce referrals of patients with a higher calculated risk score.

\section{TECHNIQUE FOR PEA}

The principle and aim of the operation is the simple removal of obstructive material to immediately reduce PVR. Like many other major operations, there are no randomised controlled trials (RCTs) to prove efficacy and the majority of the evidence for the effectiveness of the procedure is based predominantly on case series with in-hospital outcome data alone [6].

The history of the development of the current technique is interesting. Initial operative approaches in the 1950s had been unilateral by thoracotomies or transverse "clamshell" incisions and cardiopulmonary bypass (CPB) was not employed. It is likely that the first successful true endarterectomy via sternotomy (with CPB standby) was performed in 1962 by Houk et al. [8]. The first successful true endarterectomy (right side only) using CPB, via thoracotomy at UCSD was in 1970, was reported by MOSER and BRAUNWALD [9] in 1973. By 1980, DAILY et al. [10] reported the UCSD experience of three patients with a sternotomy, CPB and deep hypothermic circulatory arrest (DHCA). A review in 1984 found only 85 reported surgical cases worldwide, but by 2003, JAMIESON et al. [6] reported 1,500 cases from UCSD, emphasising the importance of sternotomy, CPB and DHCA. In the 1990s, larger programmes were also developed in Europe, initiated by $\mathrm{P}$. Dartevelle in France, E. Mayer in Germany, J. Dunning in the UK and A.M. D'Armini in Italy.
The reason the operation is difficult to perfect is related to the anatomy and physiology: location of pulmonary arteries, $5 \mathrm{~L} \cdot \mathrm{min}^{-1}$ blood flow, thin vessel wall, and a dual circulation with bronchial collateral blood flow. The basic surgical technique needed to overcome these obstacles is: median sternotomy incision for approach to both lungs; CPB to divert blood flow from the pulmonary artery and sustain function of heart and lungs; arteriotomy incisions within the pericardium and a true endarterectomy with meticulous full distal dissection to segmental vessels; and a means of reducing collateral blood flow to allow the latter [11-13]. However, despite full CPB, in many cases collateral blood flow from bronchial circulation obscures the operative field. The standard method to overcome this is full arrest of circulation, necessitating body cooling to $20^{\circ} \mathrm{C}$ to reduce metabolic activity and protect vital organ function. Thus, there is a trade-off between operative view and time for dissection against brain ischaemia during DHCA.

The technique described above has been used over the last 30 yrs in approximately 5,000 patients in total. However, some questions remain. Can the standard operation be improved? Is systemic cooling to $20^{\circ} \mathrm{C}$ really necessary? Can DHCA be reduced or avoided? Finally, is a full endarterectomy possible without DHCA? There is some experience of alternative techniques with four published reports (all from Europe) of PEA surgery without full DHCA at $20^{\circ} \mathrm{C}$. In the first publication, 30 patients operated over a 2-yr period at Hannover, Germany, were treated with an aortic occlusion balloon to occlude bronchial arteries and allow antegrade cerebral perfusion (ACP) instead of DHCA at $29.5^{\circ} \mathrm{C}$ [14]. The clinical outcome was acceptable for the era, but there was no evidence of superiority to the standard DHCA technique and no detailed cognitive function was presented. The next publication presented pooled experience, over 1 yr from Hannover, Germany, and Barcelona, Spain [15]. 30 patients were cooled to $28^{\circ} \mathrm{C}, 30^{\circ} \mathrm{C}$ or $32^{\circ} \mathrm{C}$. Moderate hypothermic circulatory arrest was employed with a mean time of $10.3 \mathrm{~min}$ in two periods. There was evidence of transient neurological dysfunction in three patients. This approach was criticised by JAMIESON [16] at the time, with concern over moderate hypothermic circulatory arrest and completeness of the endarterectomy. More recently there was a report from Papworth Hospital (Cambridge, UK) describing PEA with an attempt to avoid complete circulatory arrest, by maintaining $\mathrm{ACP}$, but with full cooling to $20^{\circ} \mathrm{C}$ [17]. 150 patients were treated over 3 yrs. ACP was achieved by aortic arch clamping to control bronchial collateral blood flow. However, in $9 \%$ of cases, DHCA (21 $\mathrm{min}$ ) was required to complete dissection adequately. Again there was no evidence of any superior outcome to the DHCA standard. In the final report, from Bologna, Italy, PEA was performed with left heart suction and moderate hypothermia instead of DHCA [18]. There were only 30 consecutive patients, collected over a 3-yr period. PEA was possible with this method, but DHCA was necessary in one patient and there was no evidence of benefit.

Alternative approaches have not yet demonstrated any benefit for patients. Therefore, new techniques should be compared with the standard, preferably in an RCT.

\section{THE BRAIN DURING PEA SURGERY}

Although the period of DHCA to allow PEA is controlled, and the majority of patients appear to have no neurological deficit 
following surgery, detailed cognitive assessments have not been made until recently. A Medline search in June 2011 with the words "pulmonary, endarterectomy, neuro" or "pulmonary, endarterectomy, cognitive" resulted in only a single publication [19]. A search of reference lists from all other PEA related publications produced two more $[20,21]$ with evidence of brain injury, possibly related to prolonged DHCA periods. The group at Papworth Hospital, Cambridge had, therefore, become interested in brain protection during PEA surgery.

This shortage of data was the rational for the PEACOG (PEA and COGnition) trial [22], as there is potential for subtle cognitive impairment with brain ischaemia during DHCA. In aortic surgery there is a growing trend to avoid DHCA by using ACP so that the brain is always perfused. The PEACOG trial was a blinded, randomised trial directly comparing ACP and DHCA for PEA surgery. The primary outcome was change in cognitive function at 3 months and 1 yr. A standardised CPB technique was used to maximise brain protection in both groups, see below. Prednisolone at $1 \mathrm{mg} \cdot \mathrm{kg}^{-1}$ is administered pre-surgery with a propofol anaesthetic and near infra-red spectrometry (NIRS) monitoring. Cooling on CPB to $20^{\circ} \mathrm{C}$ is carefully controlled with nasopharyngeal and bladder temperature monitoring. The peripheral and central temperature differential is kept at $<5^{\circ} \mathrm{C}$. pH stat is used for cooling to allow increased vasodilatation and a low temperature gradient, and conventional alpha stat for rewarming. A head cooling jacket is applied and $1 \mathrm{~g}$ of thiopentone is administered prior to DHCA. Arrest periods are limited to $20 \mathrm{~min}$ with $>10 \mathrm{~min}$ reperfusion between arrest periods. For ACP, the aorta is clamped distal to the left carotid artery to reduce bronchial collateral flow to the pulmonary circulation but maintain CPB flow to the head and right arm, with right radial pressure monitoring. In summary, the PEACOG study demonstrated no difference between techniques for the first and all second outcomes. Cognitive function was identical in both groups post-surgery and had actually improved by 3 months (fig. 2). There was no evidence of cognitive impairment. The haemodynamic improvement was also equal in both groups. PEACOG is the first RCT of DHCA against $\mathrm{ACP}$, the largest RCT involving DHCA in adults, and importantly the first detailed evaluation of cognitive function following PEA. It concluded that the standard PEA operation with DHCA at $20^{\circ} \mathrm{C}$ produces reproducible excellent results for the lungs and the brain. Therefore, surgeons, and referring physicians, can be reassured that with careful anaesthetic and CPB management, DHCA for PEA is safe and well tolerated.
DHCA is technically simpler than ACP, and in the trial nine patients had to cross over to the DHCA group to allow a full distal endarterectomy, therefore full DHCA is now advocated as the method of choice for PEA surgery.

\section{AN ALTERNATIVE APPROACH TO DHCA AND BRAIN PROTECTION}

Differing techniques of circulatory arrest have recently been reported in the study by MORSOLINI et al. [23]. Since the start of their experience in 1994, 347 PEA procedures have been completed using an evolving cerebral protection strategy so there were four cohorts of patients. In the first group, PEA had been performed with conventional 20-min periods of DHCA at $20^{\circ} \mathrm{C}$, in the second group with intermittent $10-15$ min periods of DHCA with NIRS monitoring, evolving to moderate hypothermia in the third groups and multiple intermittent periods of arrest of only $7 \mathrm{~min}$ with moderate hypothermia in the fourth group. Uniquely, they did not use cardioplegic arrest to protect the heart, but relied on the short periods of circulatory arrest with frequent reperfusion. The most contemporary group treated with multiple short periods of circulatory arrest under moderate hypothermia had a lower initial PVR than the earlier cohorts, but were older. They were more likely to have an uncomplicated recovery with a trend towards reduced neurological events, but mortality was not significantly different between the four groups despite the increasing experience over the different era studies. They had concluded that their technique was safe despite very long cumulative circulatory arrest times and the fact that the extra dissection time allowed more distal disease to be cleared. However, other expert opinion is divided and many consider that although the technique is successful in their hands, it may not be easy to replicate by others, and some remain concerned about the long cumulative arrest times.

\section{FURTHER CONTROVERSIES IN PRACTICE}

Some areas of treatment in PEA surgery have little evidence base and practice varies. A recent survey of PEA surgeons was conducted to evaluate use of inferior vena cava filters, necessity of tricuspid valve repair and closure of patent foramen ovale.

There are guidelines for the use of inferior vena cava filters from haematologists [24]. The indications for filter insertion include CTEPH. Complications are rare, but potential immediate complications include misplacement of the vena cava filter $(1.3 \%)$, pneumothorax $(0.02 \%)$, haematoma $(0.6 \%)$, air embolism $(0.2 \%)$, carotid artery puncture $(0.04 \%)$ and arteriovenous
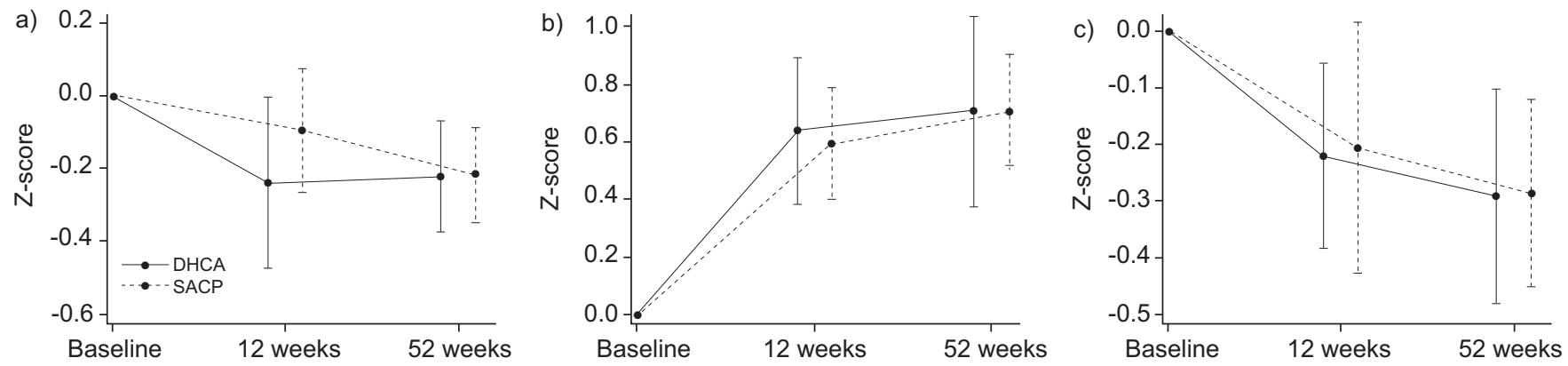

FIGURE 2. Cognitive function post pulmonary endarterectomy at baseline (pre-surgery) and at 12 and 52 weeks after surgery for a) trailmaking test, b) Rey auditory test and c) grooved peg board test. DHCA: deep hypothermic circulatory arrest; SACP: selective antegrade cerebral perfusion. Data are presented as mean (95\% CI). 
fistula $(0.02 \%)$. Potential later complications include: recurrent deep venous thrombosis $(21 \%)$, inferior vena cava thrombosis (2-10\%), post-thrombotic syndrome (15-40\%), inferior vena cava penetration $(0.3 \%)$, filter migration $(0.3 \%)$, filter tilting and fracture and entrapment of guide wires. There have been no RCT to support the use of inferior vena cava filters in patients with CTEPH and the level of evidence for recommending the use of inferior vena cava filters is low. The survey of PEA surgeons revealed mixed practice with an almost 50:50 distribution of filter use (never: one centre; rarely: five centres; frequently: no centres; always: 11 centres).

The need to repair the tricuspid valve is less controversial and expert opinion suggests that if the regurgitation is secondary to $\mathrm{CTEPH}$ alone, then repair is usually unnecessary, as postoperative right ventricular remodelling reduces regurgitation.

In the survey three respondents indicated that they always closed a patent foramen ovale, whilst three indicated that they rarely did. Reasons cited for definite closure included, previous paradoxical embolus, significant hypoxia at rest with large shunt, significant desaturation at the time of 6-min walk distance and flapping of aneurysmal septum. If there was expected severe residual $\mathrm{PH}$ then some suggest a patent foramen ovale should be left open.

\section{DIFFERENTIAL DIAGNOSIS OF CTEPH}

Careful evaluation of imaging is important because pulmonary artery obstruction is not always due to CTEPH, with pulmonary arterial hypertension (PAH)/chronic obstructive pulmonary disease with central thrombus, pulmonary artery sarcoma, fibrosing mediastinitis, pulmonary veno-occlusive disease, pulmonary vein stenosis, extrinsic vascular compression, large vessel pulmonary vasculitis, and congenital branch stenosis all featuring in the differential diagnosis. Some of these other conditions may also be treated by PEA.

\section{POST-OPERATIVE CARE}

Patients undergoing PEA are subject to many of the same post-operative complications of other cardiothoracic surgical procedures, such as atelectasis, pleural effusion, pericardial effusion, diaphragmatic dysfunction and dysrhythmias. However, reperfusion pulmonary oedema and residual $\mathrm{PH}$ are unique complications seen in the PEA patient and are associated with increased mortality.

Hypoxia is especially common in the PEA population for a number of reasons. Atelectasis is almost universal following the prolonged CPB time required for this procedure and postoperative pain and respiratory muscle dysfunction also contribute. $V^{\prime} / Q^{\prime}$ mismatch is more pronounced in the PEA patient population compared to other cardiothoracic surgery patients, presumably due to the loss of hypoxic vasoconstriction and the "steal" phenomenon which is so common following PEA. PEA causes reperfusion of lung regions by alleviating obstruction of the pulmonary arteries, resulting in the reduction of resistance in those previously obstructed vessels. If the resistance in the endarterectomised lung is lower than that of the previously patent vascular bed (lung not obstructed by chronic clot), blood flow will take the path of least resistance resulting in a relative "hyperperfusion" of newly endarterectomised lung with "steal" from previously normally perfused lung regions (fig. 3) [25]. This creates high and low $V^{\prime} / Q^{\prime}$ regions, with the low $V^{\prime} / Q^{\prime}$ regions, the newly reperfused lung regions, contributing to hypoxaemia. The redistribution of flow resolves over weeks to months resulting in more homogenous perfusion of the lung by $1 \mathrm{yr}$ [26] and most patients can be weaned off supplemental oxygen after several months.

Reperfusion oedema occurs in $10-40 \%$ of patients depending upon the definition used. It is a high permeability oedema that occurs in regions that have been endarterectomised and reperfused [27, 28]. In its most severe forms it can present as severe alveolar haemorrhage with profound hypoxaemia and is associated with a high mortality [29]. Reperfusion oedema is an early post-operative complication with $60 \%$ of cases presenting immediately following surgery, 30\% developing within the first $48 \mathrm{~h}$ post-operatively, and the minority $(10 \%)$ occurring later during the hospitalisation $(>48 \mathrm{~h})$. The incidence of reperfusion pulmonary oedema has decreased over time for unclear reasons. The definition of reperfusion pulmonary oedema in practice is arterial oxygen tension/ inspiratory oxygen fraction $<300$, opacity on a chest radiograph in a region reperfused following PEA, and no other reason for the previous two criteria (such as pneumonia or atelectasis). Severity of pre-operative $\mathrm{PH}$ and the presence of residual $\mathrm{PH}$ are associated with and increased risk of developing reperfusion pulmonary oedema [30].

The development of reperfusion pulmonary oedema is associated with a statistically significant increase in duration of mechanical ventilation and length of ICU stay [31]. Several interventions have been attempted to reduce the incidence of reperfusion lung injury. A small, two-centre clinical trial demonstrated that a strategy of minimising inotropes and low tidal ventilation following PEA could reduce the incidence of reperfusion pulmonary oedema in PEA patients as well as decrease mortality [32]. A more recent study carried out at a single institution comparing large versus small tidal volume ventilation in the PEA population is nearing completion and may provide further insight into the role of mechanical ventilation in the development of lung injury. In addition to elevated protein levels, increased neutrophils have been found in the bronchoalveolar lavage of patients with lung injury compared to those without lung injury suggesting that the injury is a consequence of inflammation [28, 33]. It is clear that larger, multicentre randomised trials are required to identify strategies to reduce reperfusion pulmonary oedema following PEA.

Treatment of reperfusion pulmonary oedema is primarily supportive. Diuresis to reduce lung water and avoidance of high cardiac output are typically employed along with minimising oxygen consumption. In contrast to patients with advanced acute respiratory distress syndrome, reperfusion pulmonary oedema can be quite asymmetric and patients may exhibit trepopnea [34], oxygenating significantly better in one lateral decubitus position versus the contralateral position. Inhaled nitric oxide may improve oxygenation and extracorporeal membrane oxygen support can be lifesaving in severe cases of reperfusion pulmonary oedema $[35,36]$.

Residual PH remains a significant problem in $5-35 \%$ of patients undergoing PEA $[6,36-38]$ and is the most common 

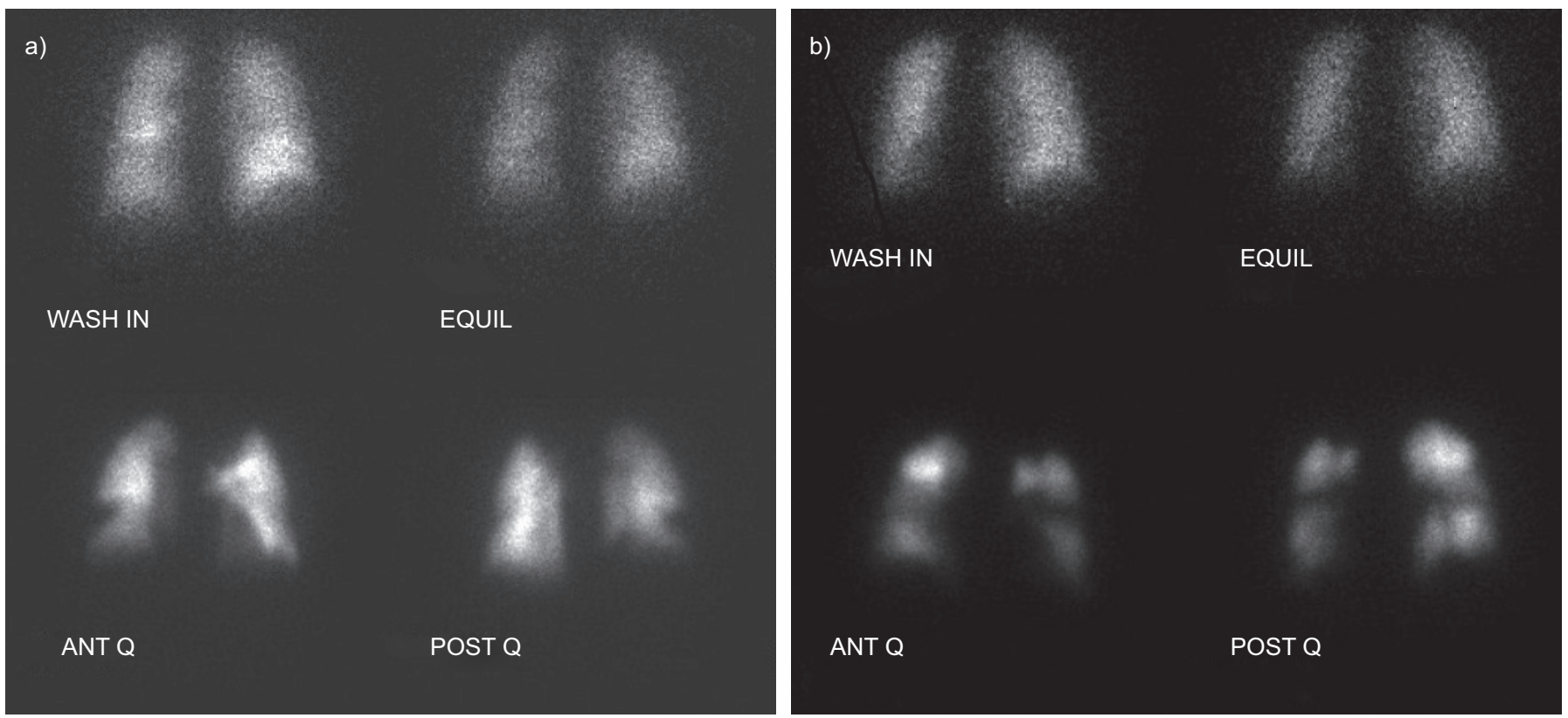

FIGURE 3. a) Pre-operative ventilation perfusion scan showing decreased perfusion to the right upper lobe, absence of perfusion to the basilar segmets of the right lower lobe, and segmental defects in the right middle lobe and left upper lobe. b) Post-operative ventilation perfusion scan with increased perfusion to the right upper and lower lobes with "steal" from the left lung. Equil: equilibration; Ant Q: anterior perfusion; Post Q: posterior perfusion.

cause of perioperative mortality at many centres [5, 7]. Operative mortality increases with increasing pre-operative PVR [5, 6], but an even greater predictor of post-operative mortality is the presence of significant residual $\mathrm{PH}$ following PEA [6, 7]. Reversible post-operative pulmonary vasoconstriction can occur as a result of $\mathrm{CPB}$, mechanical injury, and ischemia-reperfusion injury resulting in reduced levels of nitric oxide and prostacyclin and increased levels of endothelin. This condition usually resolves within $72 \mathrm{~h}[39,40]$. Irreversible PH is the result of distal chronic thromboembolic disease or superimposed small vessel vasculopathy that is not cured by the endarterectomy. Microscopically these changes appear similar to those seen in patients with PAH [41]. Determining the presence of small vessel arteriopathy prior to surgery remains problematic, but of great interest to a number of investigators $[42,43]$.

Treatment of severe residual $\mathrm{PH}$ and right heart failure in the immediate post-operative period can be challenging. Inotropic support to improve right ventricular function is usually required and careful attention to optimising right ventricular preload is important. Attempts to reduce the pulmonary artery pressure with vasodilators are often unsuccessful and carry significant risk since they can be associated with systemic hypotension. It is important to avoid hypotension and reduced perfusion of the right coronary artery that can result in worsening right ventricular function. Reduction in PVR can be obtained in some patients with inhaled nitric oxide or inhaled iloprost [40, 44, 45] without an associated reduction in systemic blood pressure. Typically, patients being treated with PAH-specific therapies (prostacyclin analogues, phosphodiesterase-5 inhibitors and endothelin receptor antagonists) prior to surgery can have these medications discontinued in the operating room if PEA has resulted in a significant reduction in PVR. However, if patients have significant residual $\mathrm{PH}$, these medications may be continued in the post-operative period once the patient is haemodynamically stable. The impact of these therapies in the early post-operative setting is unclear, mostly anecdotal, and needs to be studied. The approach to patients with milder residual $\mathrm{PH}$ following $\mathrm{PEA}$ varies according to centre. Some will begin or resume $\mathrm{PH}-$ specific therapies prior to discharge from the hospital. Others, recognising that there can be ongoing improvement in haemodynamics after surgery that appears to stabilise at 3 months following surgery [46], elect to withhold PAHspecific therapy and reassess the need for medical therapy $3-$ 6 months following surgery by echocardiography and/or right heart catheterisation. A prospective study comparing the two approaches is needed to assess how and when to best treat milder forms of residual $\mathrm{PH}$ following PEA. A recent study demonstrated that at 3 months following PEA, 31\% of patients had residual $\mathrm{PH}$ (defined as mean pulmonary artery pressure $>30 \mathrm{mmHg}$ at right heart catheterisation). However, despite worse symptoms and exercise capacity than those without residual $\mathrm{PH}$, prognosis was not affected with both groups of patients having a similar 5-yr survival [47].

Post-operative pericardial effusions are not uncommon and should be considered when patients present with worsening oedema, chest pain and dyspnoea. Echocardiography should be performed immediately to confirm the diagnosis followed by drainage of any effusion causing haemodynamic compromise. Atrial dysrhythmias are treated in a similar fashion to other patients who develop atrial flutter/fibrillation following cardiac surgery with the caveat that $\beta$-blockers are not well tolerated in patients with significant residual post-operative $\mathrm{PH}$. Anticoagulation is typically started the night of surgery in the absence of bleeding complications. Lifelong anticoagulation is recommended for all patients to avoid recurrent thrombosis. 


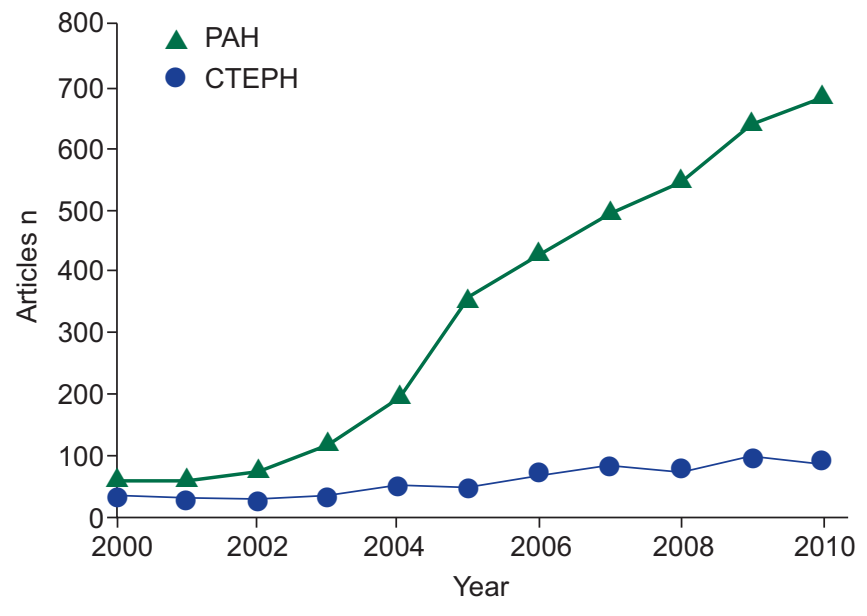

FIGURE 4. PubMed citations for chronic thromboembolic pulmonary arterial hypertension (CTEPH; $\mathrm{n}=651$ ) compared with citations for pulmonary arterial hypertension (PAH; $\mathrm{n}=3,640$ ) from 2000 to 2010.

\section{OVERVIEW AND FUTURE PERSPECTIVE}

CTEPH and its recognition and treatment have become global endeavours. Despite the growth within this field, the pace lags behind that seen in PAH over the last decade. A Medline search conducted in June 2011, with limits "Human" and search terms "Chronic Thromboembolic Pulmonary Hypertension" and "Pulmonary Arterial Hypertension" produced the following results over the past decade: CTEPH citations totalling 651, with PAH citations totalling 3,640 (fig. 4). This is not entirely surprising given the difference in diseases and interest in expanding treatment options for a chronic disease (PAH). However, the overall increase in $\mathrm{PH}$ awareness and activity globally is a reminder to ensure that this potentially curable form of $\mathrm{PH}(\mathrm{CTEPH})$ is recognised and properly treated. Given the limitations of operability assessment and role of medical therapy, some experts have suggested a PEA centre bestpractice guideline incorporating centre mortality, centre volume and capability to perform segmental endarterectomy.

\section{STATEMENT OF INTEREST}

Statements of interest for D.P. Jenkins and E. Mayer can be found at www.erj.ersjournals.com/site/misc/statements.xhtml

\section{REFERENCES}

1 Pengo V, Lensing $\mathrm{AW}$, Prins $\mathrm{MH}$, et al. Incidence of chronic thromboembolic pulmonary hypertension after pulmonary embolism. N Engl J Med 2004; 350: 2257-2264.

2 Thistlethwaite PA, Mo M, Madani MM, et al. Operative classification of thromboembolic disease determines out-come after pulmonary endarterectomy. J Thorac Cardiovasc Surg 2002; 124: 1203-1211.

3 Tunariu N. Ventilation-perfusion scintigraphy is more sensitive than multidetector CTPA in detecting chronic thromboembolic pulmonary disease as a treatable cause of pulmonary hypertension. J Nucl Med 2007; 48: 680-684.

4 Hoey ET, Mirsadraee S, Pepke-Zaba J, et al. Dual-energy CT angiography for assessment of regional pulmonary perfusion in patients with chronic thromboembolic pulmonary hypertension: initial experience. AJR Am J Roentgenol 2011; 196: 524-532.
5 Dartevelle P, Fadel E, Mussot S, et al. Chronic thromboembolic pulmonary hypertension. Eur Respir J 2004; 23: 637-648.

6 Jamieson SW, Kapelanski DP, Sakakibara N, et al. Pulmonary endarterectomy: experience and lessons learned in 1,500 cases. Ann Thorac Surg 2003; 76: 1457-1462.

7 Mayer E, Jenkins D, Lindner J, et al. Surgical management and outcomes of patients with chronic thromboembolic pulmonary hypertension: results from an international prospective registry. J Thorac and Cardiovasc Surg 2011; 141: 702.

8 Houk VN, Hufnagel CA, McClenathan JE, et al. Chronic thrombotic obstruction of major pulmonary arteries. Report of a case successfully treated by thromboendarterectomy and a review of the literature. Am J Med 1963; 35: 269-282.

9 Moser KM, Braunwald NS. Successful surgical intervention in severe chronic thromboembolic pulmonary hypertension. Chest 1973; 64: 29-35.

10 Daily PO, Johnston GG, Simmons CJ, et al. Surgical management of chronic pulmonary embolism: surgical treatment and late results. J Thorac Cardiovasc Surg 1980; 79: 523-531.

11 Berman M, Pavlushkov E, Abraham E, et al. Pulmonary endarterectomy - an example of treatment of right ventricular after load failure. MMCTS 2009; 0621: 1-8.

12 Madani M, Jamieson S. Operative techniques in thoracic and cardiac surgery 2006; 264-274.

13 Jamieson SW, Kapelanski DP. Pulmonary endarterectomy. Curr Prob Surg 2000; 37: 165-252.

14 Hagl C, Khaladj N, Peters $\mathrm{T}$, et al. Technical advances of pulmonary thromboendarterectomy for chronic thromboembolic pulmonary hypertension. Eur J Cardiothor Surg 2003; 23: 776-781.

15 Macchiarini P, Kamiya H, Hagl C, et al. Pulmonary endarterectomy for chronic thromboembolic pulmonary hypertension: is deep hypothermia required? Eur J Cardiothor Surg 2006; 30: 237-241.

16 Jamieson. Editorial comment. Eur J Cardiothorac Surg 2006; 30: 241-243.

17 Thomson B, Tsui S, Dunning J, et al. Pulmonary endarterectomy is possible and effective without the use of complete circulatory arrest - the UK experience in over 150 patients. Eur J Cardiothoc Surg 2008; 33: 157-163.

18 Mikus P, Mikus E, Martìn-Suàrez S, et al. Pulmonary endarterectomy: an alternative to circulatory arrest and deep hypothermia: mid-term results. Eur J Cardiothor Surg 2008; 34: 159-163.

19 Kunihara T, Tscholl D, Langer F, et al. Cognitive brain function after hypothermic circulatory arrest assessed by cognitive P300 evoked potentials. Eur J Cardiothor Surg 2007; 32: 507-513.

20 Wragg RE, Dimsdale JE, Moser KM, et al. Operative predictors of delirium after pulmonary thromboendarterectomy. A model for postcardiotomy delirium? J Thorac Cardiovasc Surg 1988; 96: 524529.

21 Surie S, Tijssen MA, Biervliet JD, et al. Chorea in adults following pulmonary endarterectomy. Mov Disord 2010; 25: 1101-1104.

22 Vuylsteke A, Sharples L, Charman G, et al. Circulatory arrest versus cerebral perfusion during pulmonary endarterectomy surgery (PEACOG): a randomized controlled trial. Lancet 2011; 378: 1379-1387.

23 Morsolini M, Nicolardi S, Milanesi E, et al. Evolving surgical techniques for pulmonary endarterectomy according to the changing features of chronic thromboembolic pulmonary hypertension patients during 17-year single-center experience. J Thorac Cardiovasc Surg 2012; 144: 100-107.

24 British Committee for Standards in Haematology Writing Group, Baglin TP, Brush J, et al. Guidelines on the use of vena cava filters. Br J Haematol 2006; 134: 590-595.

25 Olman MA, Auger WR, Fedullo PF, et al. Pulmonary vascular steal in chronic thromboembolic pulmonary hypertension. Chest 1990; 98: 1430-1434.

26 Moser KM, Metersky ML, Auger WR, et al. Resolution of vascular steal after pulmonary thromboendarterectomy. Chest 1993; 104: 1441-1444. 
27 Levinson RM, Shure D, Moser KM. Reperfusion pulmonary edema after pulmonary artery thromboendarterectomy. Am Rev Respir Dis 1986; 134: 1241-1245.

28 Kerr KM, Auger WR, Marsh JJ, et al. The use of cylexin (CY-1503) in prevention of reperfusion lung injury in patients undergoing pulmonary endarterectomy. Am J Respir Crit Care Med 2000; 162: $14-20$.

29 Manecke GR, Kotzur A, Atkins G, et al. Massive hemorrhage after pulmonary endarterectomy. Anesth Analg 2004; 99: 672-675.

30 Kerr KM, Auger SR, Marsh JJ, et al. Efficacy of methylprednisolone in preventing lung injury following pulmonary thromboendarterectomy. Chest 2012; 141: 27-35.

31 Duwe BV, Kerr KM, Fedullo PF, et al. Clinical imparct of reperfusion lung injury on patients undergoing pulmonary thromboendarterectomy. Am J Respir Crit Care Med 2009; 179: A4628.

32 Mares P, Gilbert TB, Tschernko EM, et al. Pulmonary artery thromboendarterectomy; a comparison of two different postoperative treatment strategies. Anesth Analg 2000; 90: 267-273.

33 Auger WR, Moser KM, Comito RM, et al. Efficacy of intravenous ICI 200,880 in the prevention of adult respiratory distress syndrome in patients undergoing pulmonary endarterecomy. Am J Respir Crit Care Med 1994; 149: A1032.

34 Wood FC. Trepopnea. Arch Intern Med 1959; 104: 966-973.

35 Berman M, Tsui S, Vuylsteke A, et al. Successful extracorporeal membrane oxygenation support after pulmonary thromboendarterectomy. Ann Thorac Surg 2008; 86: 1261-1267.

36 Thistlethwaite PA, Madani MM, Kemp AD, et al. Venovenous extracorporeal life support after pulmonary endarterectomy: indications, techniques and outcomes. Ann Thorac Surg 2006; 82: 2139-2146.

37 Thistlethwaite PA, Kaneko K, Madani MM, et al. Technique and outcomes of pulmonary endarterectomy surgery. Ann Thorac Cardiovasc Surg 2008; 14: 274-282.
38 Bondermann D, Skoro-Sager N, Jakowitsch J, et al. Predictors of outcome in chronic thromboembolic disease. Circulation 2007; 115: 2153-2158.

39 Riedel B. The pathophysiology and management of perioperative pulmonary hypertension with specific emphasis on the period following cardiac surgery. Int Anesthesiol Clin 1999; 37: 55-79.

40 Kramm T, Eberle B, Guth S, et al. Inhaled iloprost to control residual pulmonary hypertension following pulmonary endarterectomy. Eur J Cardiothoracic Surg 2005; 28: 882-888.

41 Moser KM, Bloor CM. Pulmonary vascular lesions occurring in patients with chronic major vessel thromboembolic pulmonary hypertension. Chest 1993; 103: 685-682.

42 Kim NH, Fessler P, Channick RN, et al. Preoperative partitioning of pulmonary vascular resistance correlates with early outcome after thromboendarterectomy for chronic thromboembolic pulmonary hypertension. Circulation 2004; 109: 18-22.

43 Hardziyenka M, Reesink HJ, Bouma BJ, et al. A novel echocardiographic predictor of in-hospital mortality and mid-term haemodynamic improvement after pulmonary endarterectomy for chronic thromboembolic pulmonary hypertension. Eur Heart $J$ 2007; 28: 842-849.

44 Flonder M, Merkel M, Hofsteter C, et al. The effect of inhaled nitric oxide and inhaled iloprost on hypoxaemia in a patient with pulmonary hypertension after pulmonary thromboendarterectomy. Anaesthesia 2006; 61: 1200-1203.

45 Imanaka $\mathrm{H}$, Miyano $\mathrm{H}$, Takeuchi $\mathrm{M}$, et al. Effects of nitric oxide inhalation after pulmonary thromboendarterectomy for chronic thromboembolism. Chest 2000; 118: 39-46.

46 Corsico AG, D'Armini AM, Ceveri I, et al. Long term outcome after pulmonary endarterectomy. Am J Respir Crit Care Med 2008; 178: 419-424.

47 Freed DH, Thompson BM, Berman M, et al. Survival after pulmonary thromboendarterectomy; effect of residual pulmonary hypertension. J Thoracic Cardiovasc Surg 2011; 141: 383-387. 